University of Nebraska - Lincoln

DigitalCommons@University of Nebraska - Lincoln

\title{
Optimal Foraging in a Uniform Habitat: The Search Mechanism of the Green Lacewing
}

Alan B. Bond

University of Nebraska - Lincoln, abond1@unl.edu

Follow this and additional works at: https://digitalcommons.unl.edu/bioscibond

Part of the Behavior and Ethology Commons

Bond, Alan B., "Optimal Foraging in a Uniform Habitat: The Search Mechanism of the Green Lacewing" (1980). Alan Bond Publications. 4.

https://digitalcommons.unl.edu/bioscibond/4

This Article is brought to you for free and open access by the Papers in the Biological Sciences at DigitalCommons@University of Nebraska - Lincoln. It has been accepted for inclusion in Alan Bond Publications by an authorized administrator of DigitalCommons@University of Nebraska - Lincoln. 


\title{
Optimal Foraging in a Uniform Habitat: The Search Mechanism of the Green Lacewing
}

\author{
Alan B. Bond \\ Department of Zoology, University of California, Berkeley, California 94720 \\ Present address [1980]: Department of Psychology, University of California, Berkeley, CA 94720
}

\begin{abstract}
The effects of food deprivation and prey contact on the components of searching behavior in larval green lacewings (Chrysopa carnea Stephens) were examined to test the applicability of optimal foraging theory to predation in a uniform habitat. Variation in foraging intensity was primarily the result of changes in the meander. Modulation of the response to prey contact with increasing deprivation involved changes in the velocity and the response persistence and suggested the occurrence of adaptation to inferred differences in the spatial distribution of the prey. The ratio of giving-up times at different levels of deprivation was in accordance with the predictions of optimal foraging theory, even though the conditions precluded a discrete decision process.
\end{abstract}

The theory of optimal foraging contends that predators feeding on patchily distributed prey can allocate their foraging efforts according to the pattern of availability of the resource, and thereby maximize their feeding rate, through the use of decision rules that determine the length of time to persist in exploring a given patch (Pyke et al. 1977). The model developed by Charnov (1976a) is explicitly concerned with the case in which differences in prey density are expressed as differences in the number of prey per patch, rather than the average patch area or the distance between patches. The decision rule in these circumstances requires an estimate of the mean rate of prey capture; an independent measure of the feeding rate within a particular patch; and an awareness of the predator's position in space, that is, whether it is within a patch or between patches. Given these three pieces of information, the optimal searching strategy of the predator is strictly determined (Charnov 1975, 1976b).

The assumptions concerning the estimation of the average feeding rate and the capture rate within a given patch are not unreasonable, and some support for the independence of these measures has been provided by Cowie's (1977) study of the foraging of great tits. The feature of Charnov's model that seems most artificial and anomalous, however, is the assumption of an invariant distribution of discrete, delimited prey patches, in which discovery of a patch is held to be a separate event from the discovery of prey. Realworld analogues of this situation certainly exist: one is reminded of chalcid wasps searching for hosts on individual flower heads (Varley 1941) or tits feeding on larvae concealed in pine cones (Gibb 1958). There is also no question that experimental conditions appropriate to the model can be manufactured, and that several species of insectivorous bird have been shown to be capable of foraging in these laboratory systems in a manner quite consistent with Charnov's predictions (Smith \& Sweatman 1974; Krebs et al. 1974; Zach \& Falls 1976; Cowie 1977). In many cases in the real world, however, the area of prey patches and their spacing are unlikely to be independent of prey density, and few natural demarcations exist to distinguish the area within patches from the rest of the environment (Curio 1976).

In a uniform habitat, in which the only reliable indication of the discovery of a patch is encounter with a prey item, searching within patches intergrades with searching between patches. Giving-up time is, therefore, less the result of a specific decision than the indirect consequence of gradual shifts in the components of the searching behavior (e.g. Chandler 1969; Murdie \& Hassell 1973; Smith 1974a, 1974b). Optimality criteria for foraging in these systems have not been explored, and the degree to which Charnov's model of the discrete situation can be applied to them is unknown. An analysis of the components of the searching behavior of a predator that must deal with patches of variable size and uncertain location would go far toward determining the limitations of optimal foraging theory and describing the means by which a 
continuous variation in the distribution of prey may be assessed and exploited.

When prey are patchily distributed, the searching behavior of a predator can be conventionally divided into two contrasting modes or phases. The predator first discovers a prey patch by sampling superficially over a large area; it then alters its behavior and investigates thoroughly a localized region around the point of prey contact, an activity usually described as "area-restricted" or "area-concentrated" searching (Curio 1976). To simplify reference to different search phases and to emphasize the functional contrast between them, I propose to call these behaviors 'extensive' and 'intensive' searching, respectively, after terms used to describe strategies of labor investment in agriculture. Alternation of extensive and intensive searching has been recorded in many species, but the best-documented and most conspicuous biphasic strategies occur among the guild of insects that feed predominantly on sedentary homoptera: coccinellids, syrphid flies, and lacewings (reviews in Krebs 1973; Curio 1976).

The larva of the green lacewing, Chrysopa carnea Stephens, is a widespread, economically important predator on aphids and other patchily distributed plant pests. Searching behavior in this species is easily elicited on horizontal surfaces in the laboratory (Fleschner 1950; Bänsch 1964) and is qualitatively similar to that of other aphid predators. Intensive searching in chrysopids is initiated subsequent to feeding or upon contacting prey with the mandibles. Predation is apparently not elicited by visual or olfactory cues (Fleschner 1950). Intensive searching in these animals appears to involve a reduction in velocity, an increase in the rate of turning, and an increase in the width of the search path (Bänsch 1964), although quantitative studies of the behavior have never been conducted. If no further prey are encountered, the search gradually reverts to the extensive phase.

In the open-field searching of chrysopid larvae, therefore, we have a system that is appropriate in its simplicity to Charnov's theoretical model. Arthropodan predators probably employ their level of hunger motivation as the best available estimate of the average feeding rate (Charnov 1976b; Wilson 1976), so the value of this parameter can be conveniently set by satiating the larvae and depriving them for a predetermined period of time. To assure the constancy of the estimated feeding rate, we will record only the predator's response to brief contacts with prey and not allow episodes of prolonged feeding that might change the hunger state. The blind, random search of the larva, on the other hand, guarantees that patch discovery is a consequence of prey discovery and that the distribution of searching effort in space will be entirely a projection of the temporal patterning of the search components. We can then inquire how the pattern of the search behavior determines the spatial distribution of searching effort, how it is altered with changes in feeding rate or apparent prey density, and how the resulting distribution of searching time compares with the predictions of optimal foraging theory.

\section{Methods}

Lacewing eggs (Chrysopa carnea Stephens) were obtained from a laboratory culture maintained by the University of California Agricultural Experiment Station in Albany, California. All animals used in this study were second- to tenth-generation descendants of adults captured in the field. The larvae were reared individually in $15-\mathrm{ml}$ shell vials in a controlled environment: $21.0 \pm 0.5{ }^{\circ} \mathrm{C}$, about $50 \%$ relative humidity, and a 16L:8D (long day) light cycle. Freshly killed pea aphids, Acyrthosiphon pisum (Harris), were supplied in excess once daily. Experiments were conducted on the day following the first molt, approximately seven to eight days after hatching. Variation in prey availability was simulated by manipulating the level of food deprivation. The experimental treatment consisted of confining the animals for $30 \mathrm{~min}$ in continuous contact with food to induce satiety and subsequently depriving them for either 2 or $8 \mathrm{~h}$. Earlier studies (Bond 1978b) have provided evidence of the efficacy of the satiation procedure and have demonstrated that larvae exposed to these two levels of deprivation display significantly different levels of hunger.

The searching behavior of each larva was observed in an open, two-dimensional arena, delimited by a 56 $\times 31 \mathrm{~cm}$ wooden frame clamped tightly to a tabletop. The arena surface was covered at the start of each run with a clean sheet of absorbent paper ("Bench Kote") to prevent any accumulation of odor traces and excreta, and was illuminated uniformly from above at an intensity of about $4000 \mathrm{~lx}$. The animals were restricted to the arena with a strand of 19-gauge nichrome wire, heated to about $50{ }^{\circ} \mathrm{C}$. The arena was sufficiently shallow that a thermal gradient could be detected only within about $2 \mathrm{~cm}$ of the frame.

The position of the larva in the arena was recorded indirectly by means of its reflection in a vertical pane of tinted plate glass, $0.64 \mathrm{~cm}$ thick, mounted adjacent to and paralleling the long edge of the frame (Figure 1 ). When the incident light intensities on each side of 
the pane were properly adjusted, it was possible to obtain both a reflected, virtual image of the animal and an equally bright, transmitted image of the recording surface behind the glass. The apparent movement of the image of the larva over the area behind the glass constituted an undistorted, reversed projection of the animal's behavior. The apparent position of the image was plotted on the recording surface every $1.5 \mathrm{~s}$ for the duration of the run, while the number of intervals in which no movement took place was recorded on a hand-counter. The co-ordinates of each successive point on the search path were measured to the nearest $0.25 \mathrm{~mm}$ on a Bendix Data-Grid Digitizer and recorded on tape for further analysis. A total of 90 animals were tested in this apparatus, 15 at each deprivation level during extensive searching and 30 at each level during the intensive phase.

The protocol for extensive searching involved releasing the larva gently near the center of the arena and recording its movements for $8 \mathrm{~min}$, or until at least $5 \mathrm{~min}$ of active locomotion had been observed. Because the proportion of time spent immobile varied among individuals from zero to more than $60 \%$, it was not possible to obtain equal time-in-motion sample sizes for all animals tested. When the larva approached within $2 \mathrm{~cm}$ of the wire barrier, it was picked up with a camel's-hair brush and returned to the center of the arena. As was the case in Chan- dler's (1969) study of the searching of syrphid fly larvae, gentle manipulation of the predator during the course of the run appeared to have no significant impact on its subsequent behavior.

Intensive searching was elicited by presenting to the predator the abdomen of a medium-sized pea aphid on micro-dissection forceps. When the larva's mandibles had penetrated the aphid and feeding had just commenced, the prey was gradually but firmly withdrawn, thereby minimizing intake and maintaining the motivational state of the predator. Hand-feeding in this fashion insured that at least two satisfactory intensive searching responses of perhaps 2 or 3 min duration could be obtained from each larva during its allotted $20 \mathrm{~min}$ of activity. Provided that care was taken not to alarm the animal by abrupt or forceful removal of the food, the subsequent searching behavior was qualitatively indistinguishable from that which followed feeding to completion on first-instar aphids.

\section{Results}

\section{Spatial Analysis}

The characteristic features of intensive searching in chrysopids, which include a decrease in velocity, an increase in turning rate, and an increase in the amplitude of scanning movements of the head, were

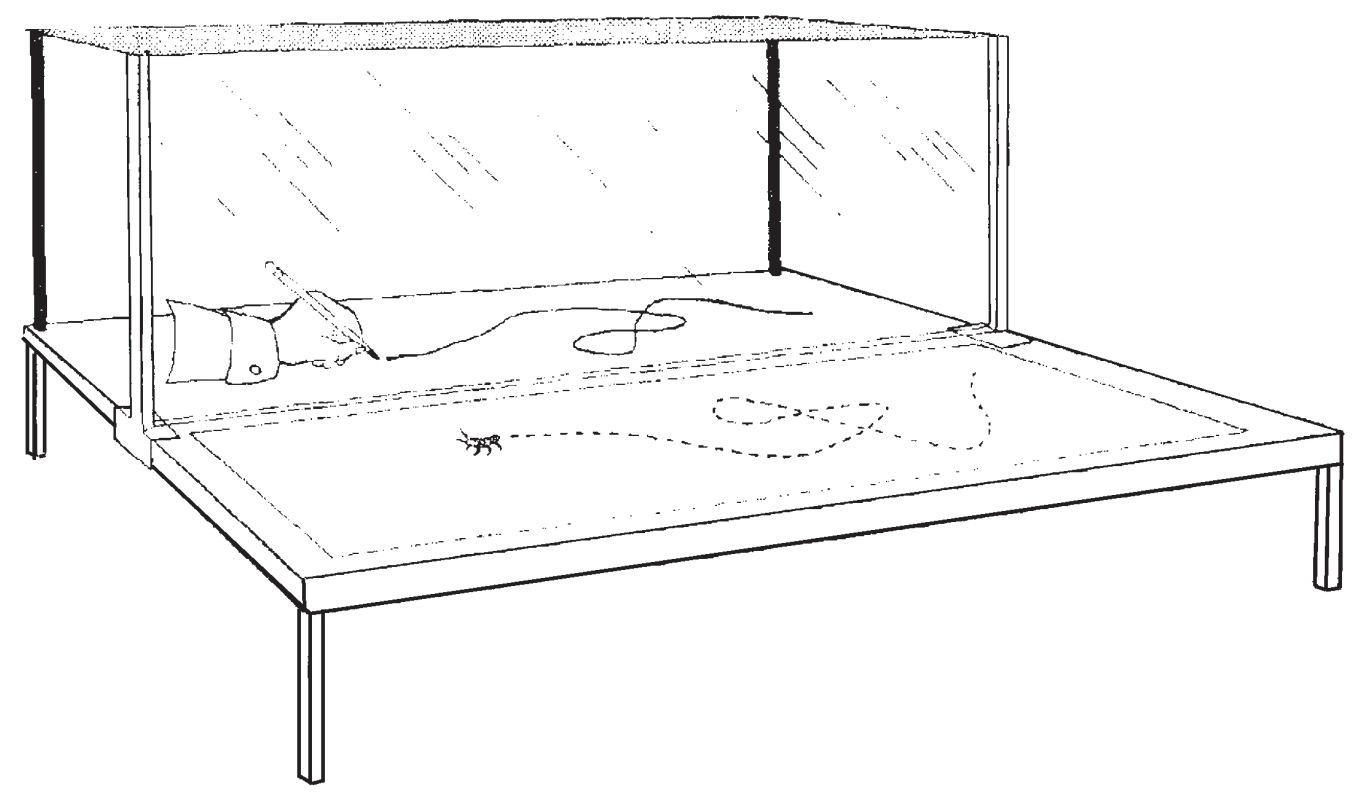

Figure 1. Apparatus for recording searching paths by tracing the movements of the animal's image. 
judged to be most conspicuously displayed within a radius of at most $2.5 \mathrm{~cm}$ from the point of prey contact, the region delimited by solid vertical lines in Figure 2. For purposes of spatial analysis, therefore, responses to prey were arbitrarily divided into "intensive" and "post-intensive" or "recovery" phases at the step at which the predator first passed beyond the 2.5-cm radial limit.

For each 1.5-s step in the search paths, the linear displacement and the magnitude of the deviation from the previous step were computed, and estimates of three variables were derived: velocity; rate of turning; and ratio of turning rate to velocity, a measure of path convolution that I have termed "meander." Search paths were then divided into segments of 20 consecutive steps to extract two grouped measures of searching pattern.

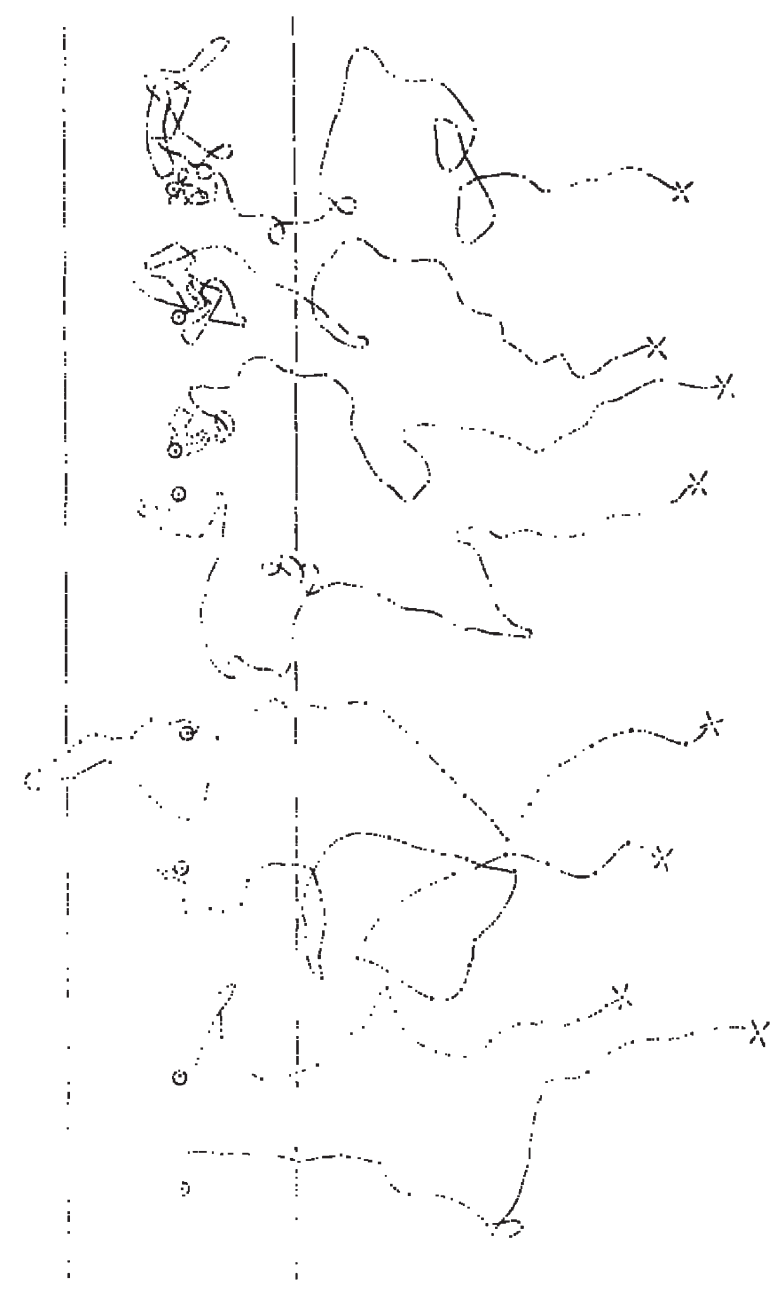

Figure 2. Intensive searching traces. Dots indicate 1.5-s intervals. $\mathrm{O}=$ start; $\mathrm{X}=$ end. Solid vertical lines are placed $2.5 \mathrm{~cm}$ from the starting point to show separation of intensive and post-intensive phases.
The turning asymmetry of each path segment was estimated, using the proportional deviation of the turning distribution from equiprobability (Bond MS). If $p$ is the proportion of right turns in the sample, then the asymmetry, $A$, is defined as

$$
A=2|p-0.5|
$$

In the absence of a bias in the distribution of turns, the expectation of $A$, given a sample size of 20 , is $1 / 2 \times \sqrt{5} \approx 0.224$.

A single measure of searching intensity that appears to incorporate the effects of both asymmetry and meander and is easily estimated from field observations is the ratio of the total length of a path segment to the linear displacement between the ends of the segment (Smith 1974b; Williamson \& Gray 1975). This measure has the disadvantage of being extremely sensitive to the sequential pattern of turning (Smith 1974b) however, yielding an unacceptably large coefficient of variation when it is applied to random searching data. Some improvement can be obtained by employing the diameter of the minimum circumscriptive circle (the smallest circle that will just enclose all points on the path), rather than the linear displacement, as the denominator (Bond MS). Neither of these measures is effective in detecting differences in searching intensity of the type observed in this study, however, since changes in velocity and turning rate that are equal in relative magnitude and opposite in sign will leave the distance ratios virtually constant. To avoid these difficulties, searching intensity was assessed using the ratio of the area searched to the area of the minimum circumscriptive circle, a measure I have called "thoroughness" (Bond MS). When the influence of asymmetry and turn sequence is negligible, the thoroughness, $T$, can be approximated as

$$
T=1-\exp \left(\frac{-4 L w}{\pi D^{2}}\right)
$$

where $L=$ path length, $w=$ path width, and $D=$ diameter of the minimum circumscriptive circle (Bond MS). Using an estimate of $2 \mathrm{~mm}$ for the path width, values of $T$ were calculated for each 20 -step path segment.

Due to the enormous differences in sample size between treatments and among animals, a betweensubjects comparison proved to be impracticable. Values for each of the five path variables from a total of 15 animals for each deprivation level and phase 
Table I. Mean Values of Path Variables

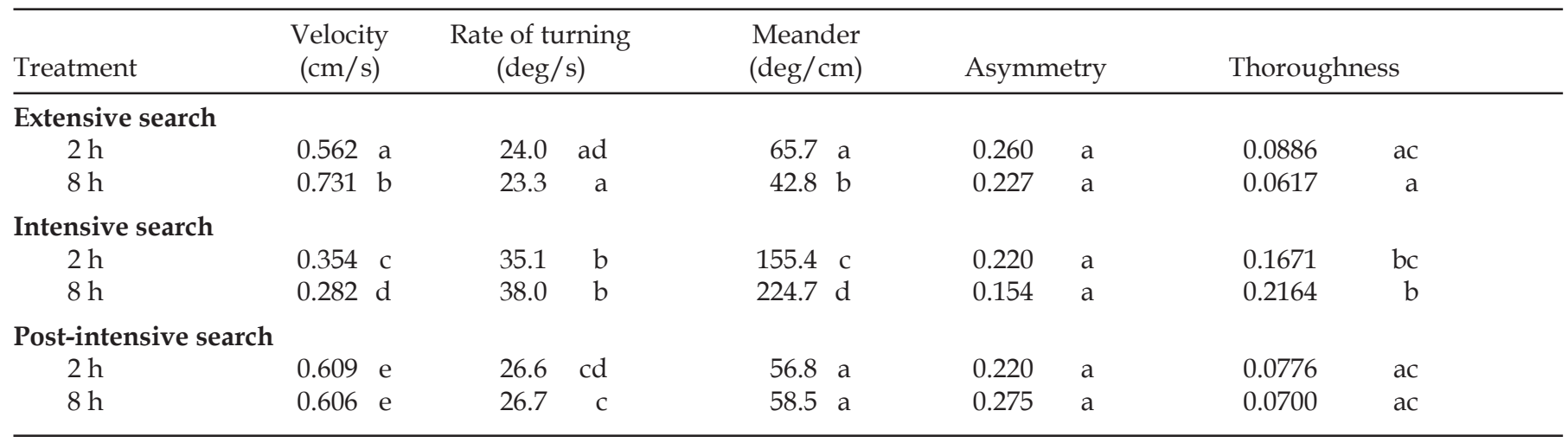

Approximate sample sizes for step variables: 2900 per treatment, extensive; 410, intensive; 930, post-intensive. Sample sizes of grouped variables were about $5 \%$ of these values. Means that do not share a common letter are significantly different $(P<0.05)$ by a modification of Scheffé's method of multiple contrasts (Marascuilo 1971). None of the means for asymmetry differ significantly from a random expectation.

of searching were therefore pooled and subjected to one-way analysis of variance with multiple planned comparisons (Table I).

\section{Temporal Analysis}

The extensive searching animals were classified as either "inactive" or "active" on the basis of whether or not the total time spent motionless exceeded $15 \%$ of the observation period. There was no apparent difference between active and inactive animals in mean velocity or turning rate. Although the proportion of inactive animals was larger at the higher deprivation level (50\% versus $26 \%$ ), the difference failed to achieve statistical significance.

There was some suggestion of temporal patterning in the occurrence of large-magnitude turns during extensive searching. A typical trace (Figure 3) shows evidence of a periodic enhancement in the rate of turning, producing bouts of spiral or looping movement about 2 to $4 \mathrm{~cm}$ in diameter. Turning within bouts was always asymmetrical, with the direction of bias remaining consistent for a given animal for the duration of the observation period.

The temporal patterning of search behavior following prey contact was analyzed by calculating the mean velocity and turning rate for each successive step during intensive searching. To describe the pattern of change in these variables, the decay function

$$
Y=\frac{a}{b+t}+c
$$

(where $t$ is the time since prey contact; $Y$ is the value of the path variable; and $a, b$, and $c$ are regression parameters) was fitted to the distributions of mean val- ues using a non-linear least-squares procedure based on Best's (1973) method of feasible conjugate direction. The average velocity and turning rate for each 6-s interval for the first 2 min following prey contact and the corresponding fitted decay curves are graphed in Figures 4 and 5.

Three descriptive parameters were derived from the coefficients of the best-fit function. The horizontal asymptote, $\mathrm{c}$, is that value of $Y$ to which the intensive searching response will decay when given unlimited time, i.e. the value of $Y$ during extensive searching.

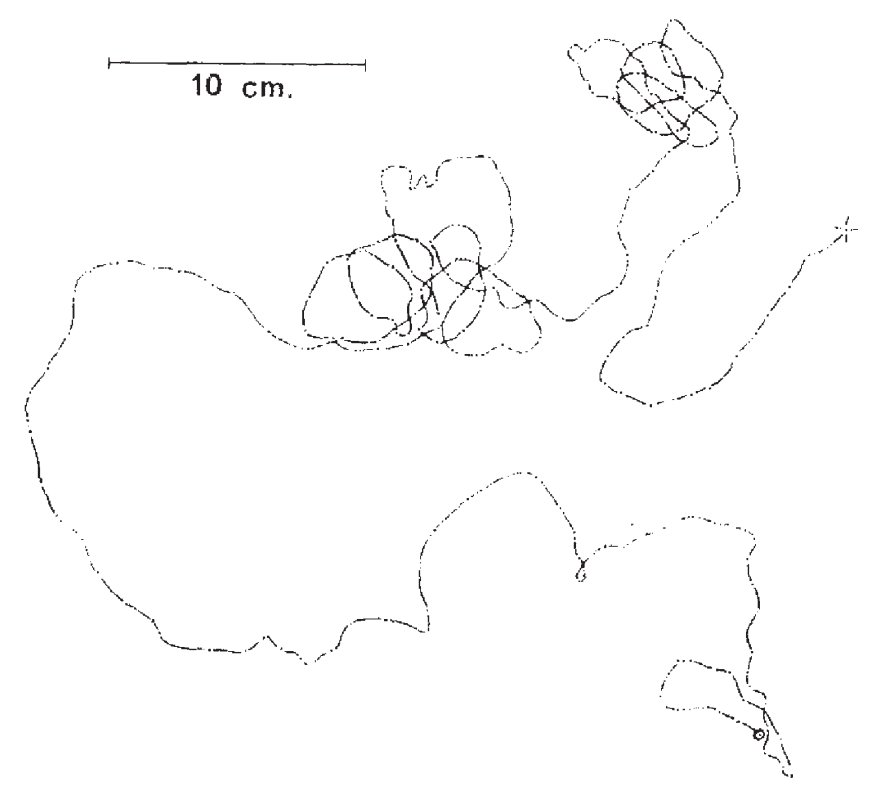

Figure 3. Extensive searching trace. Dots indicate 1.5-s intervals. $\mathrm{O}=$ start; $\mathrm{X}=$ end. 


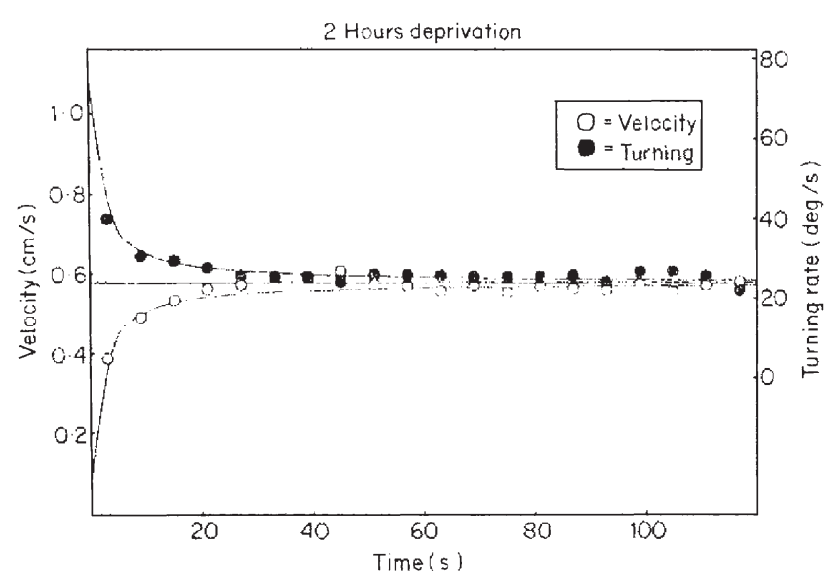

Figure 4. Mean velocity and turning rate for each 6-s interval following prey contact: 2 -h deprivation.

The $y$-intercept, $i=a / b+c$, represents the level to which $Y$ is set just subsequent to prey contact. Finally, the decay constant, $b$, is a measure of the rate at which the value of $Y$ changes over time. Estimates of these three parameters for velocity and turning rate at each deprivation level are provided in Table II.

\section{Distribution of Searching Effort}

The spatial allocation of searching effort during intensive searching was analyzed directly by converting the step sequence to polar coordinates centered on the point of prey contact. The duration of movement within each of seven concentric radial intervals, ranging from $0.58 \mathrm{~cm}$ to $4.06 \mathrm{~cm}$ in equal increments, was calculated from each path sample and subjected to statistical analysis. Means of these measures for each deprivation treatment are graphed in Figure 6. The mean duration of search in each interval was consistently greater for animals at the higher deprivation level, but this difference was statistically significant only for the $0.58-\mathrm{cm}$ interval $(P$ $<0.01$; $t$-test).

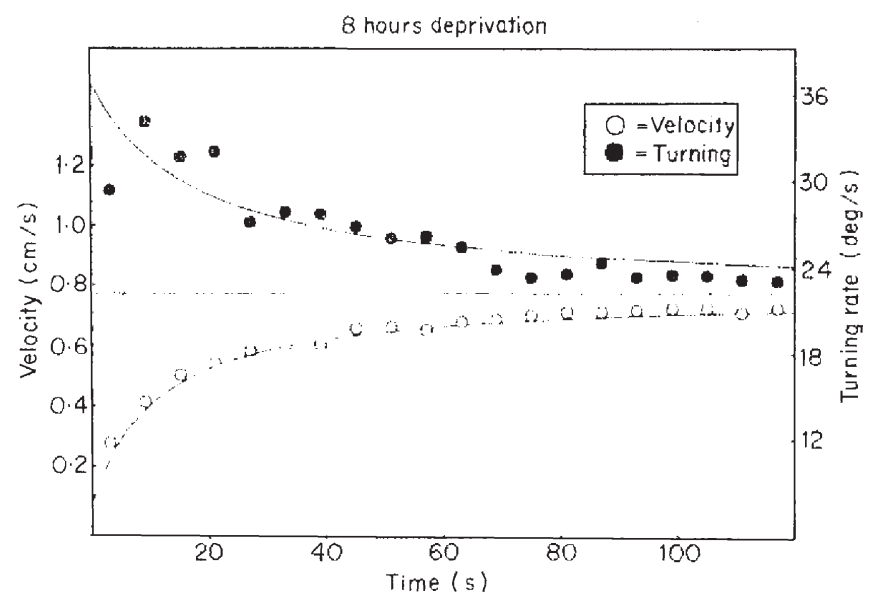

Figure 5. Mean velocity and turning rate for each 6-s interval following prey contact: 8-h deprivation.

\section{Discussion}

\section{The Search Mechanism}

Intensive searching in lacewings resembles that described for numerous other insect species (e.g. Bänsch 1964; Chandler 1969; Murdie \& Hassell 1973; Jones 1976), in that it clearly entails a decrease in velocity and an increase in the turning rate relative to the extensive phase (Table I). The results of this study suggest that these changes reflect the operation of a single underlying search mechanism, one manifestation of which is the constancy of the decay parameter within deprivation levels (Table II). The similarity in rates of decay for turning and velocity assures that the proportional differences between search phases in these variables will be of roughly similar magnitude: turning rate increased after prey contact by $46 \%$ and $63 \%$ for animals deprived for $2 \mathrm{~h}$ and $8 \mathrm{~h}$, respectively, while velocity decreased by $37 \%$ and $61 \%$ (Table I). In consequence, changes in velocity and turning appear to contribute equally to the increase in meander during intensive searching.

Table II. Best-Fit Parameters of the Decay Process for Intensive Searching

\begin{tabular}{llcccc}
\hline & \multicolumn{2}{c}{$2 \mathrm{~h}$} & & \multicolumn{2}{c}{$8 \mathrm{~h}$} \\
\cline { 2 - 3 } \cline { 5 - 6 } Parameter & Velocity & Rate of turning & & Velocity & Rate of turning \\
\hline Asymptote & 0.578 & 23.7 & & 0.768 & 22.2 \\
Intercept & 0.0814 & 72.8 & & 0.0875 & 36.8 \\
Decay constant & 1.62 & 1.73 & & 11.18 & 18.11 \\
\hline
\end{tabular}




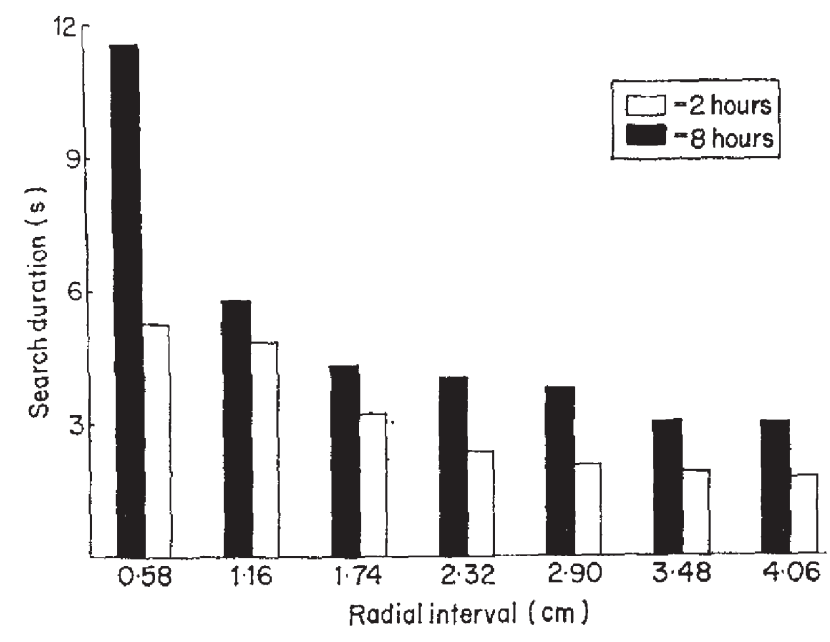

Figure 6. Duration of movement within a radial interval during intensive searching versus distance from prey contact.

Modulation of the prey response as a function of hunger level appears to be accomplished primarily through manipulation of the decay parameter and the asymptotic value of the velocity. There are no indications that turning rate during extensive searching was affected by deprivation, but the velocity was significantly higher in animals deprived for $8 \mathrm{~h}$ (Table I). The influence of deprivation on the decay parameter is strikingly illustrated in the temporal analysis graphs (Figures 4 and 5), showing that changes in velocity and turning were more persistent at the higher level of deprivation. There is some suggestion that hunger may influence the turning- rate intercept (Table II), but the observed difference could easily have arisen by chance, particularly given the degree of scatter in the turning-rate data (Figure 5).

Within the limits of this study, variation in the pattern of searching in lacewings appears to depend on changes in the meander alone. There were certainly no differences in asymmetry across treatments (Table I). The similarity in the relative magnitude of treatment effects on meander and thoroughness, furthermore, implies that changes in the sequential pattern of turning or systematic avoidance of areas previously searched do not play a significant role in this system (Table I), The only remaining variable that could conceivably affect searching intensity is path width, which unfortunately proved very difficult to quantify. The apparent increase in the amplitude of scanning movements of the head after prey contact, however, suggested that changes in path width may accentuate the effect of differences in meander during intensive searching.

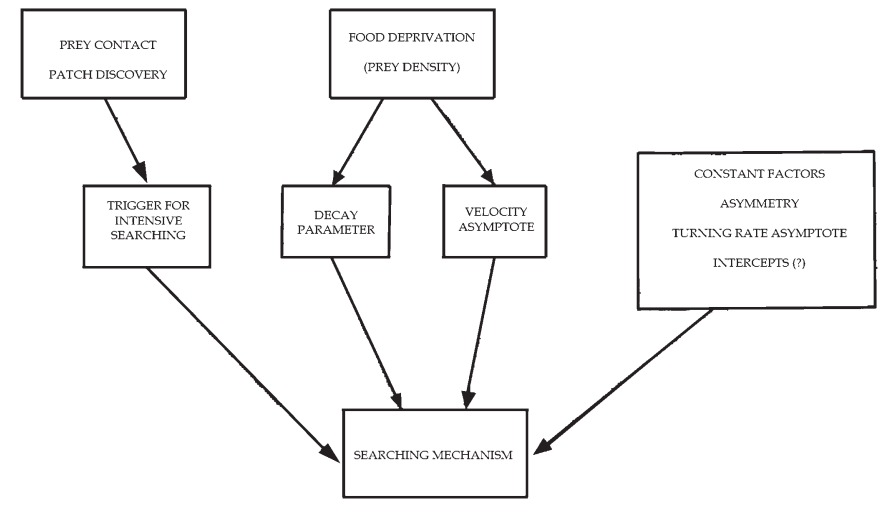

Figure 7. Diagram of hypothetical model of the search mechanism.

The behavioral model that emerges from this analysis is summarized in Figure 7. The velocity during extensive searching and the decay parameter are determined on the basis of hunger-derived estimates of feeding rate. All other factors, including turning rate and asymmetry, are presumed to remain constant. Discovery of a patch triggers a deflection of the path variables to some constant level (the intercept), followed by a gradual decay to pre-contact levels, the course of which is strictly determined by the asymptote and the decay parameter. Even if one includes the possibility of changes in the path width, the search mechanism is exceedingly simple. We must therefore address the question of whether such a simple mechanism is, in fact, sufficient to produce an optimal allocation of searching effort.

\section{The Distribution of Searching Effort}

The modulation of meander according to level of deprivation suggests the possibility that predators may tailor their search behavior to fit predictions of patch size and spacing. During extensive searching, high-deprivation animals turn at the same rate as low-deprivation animals while moving faster. The result is a lower meander and a more linear search path, consistent with the likelihood of a greater mean distance between patches when the prey density is low. The increase in meander following prey contact is enhanced at high deprivation levels by an increase in the decay parameter, producing a more compact pattern of intensive searching at low prey densities, in keeping with the likelihood of smaller prey patches. 
The direct analysis of the pattern of distribution of searching time (Figure 6) lends some support to this inference. Within the $2.5-\mathrm{cm}$ radius that delimits intensive searching, $45 \%$ of the foraging time of 8 -hdeprivation animals was spent within $0.58 \mathrm{~cm}$ of the point of prey contact. Two-hour deprivation animals covered a broader area during their search and invested only $33 \%$ of their intensive search time in this central interval. The difference was statistically significant $(P<0.05$; $t$-test $)$.

This argument for the adaptive significance of the resultant spatial distribution of searching effort is, admittedly, only speculative. To make firmer judgments would require some knowledge of the variation in patch size and spacing with density in the principal prey species. Nevertheless, it appears possible that these simple alterations of search behavior with hunger level could compensate, in some degree, for differences in spatial distribution and thereby significantly influence the rate of prey contact.

\section{The Evidence for Optimality}

The inference from Charnov's model that has achieved the status of a principal criterion for the occurrence of optimal foraging is the prediction that the giving-up time for a patch should be inversely proportional to the overall prey density or mean feeding rate and independent of the number of prey encountered within a patch (Krebs et al. 1974). Provided that an animal invests its searching effort to maximum advantage, the ratio of giving-up times at two different prey densities should then be equal to the inverse of the ratio of the densities. This proposition has been tested with considerable success in several other laboratory systems (Krebs et al. 1974; Zach \& Falls 1976). Because differences in density in this study were simulated by manipulating the hunger level of the predator, calculation of the optimum time ratio requires a few additional assumptions, but the derivation is still fairly straightforward.

The quantity of food in the gut at any given time is probably the most immediate indicator of the level of prey availability in the recent past. If we accept Holling's (1966) hypothesis that the rate of gut emptying is a linear function of the volume of gut contents, $V$, the rate of emptying should, under equilibrium conditions, come to approximate the mean feeding rate at that prey density (Charnov 1976b). The ratio of estimated prey densities at two different hunger levels can thus be assessed directly from the ratio of gut volumes. If an animal is fed to its maximum gut capacity, $K$, and then deprived for $t h$, the volume of food in the gut can be measured by the difference between $K$ and the meal size at that deprivation, $H(t)$. Meal size in Chrysopa has been shown to fit the function:

$$
H=\frac{K t}{a+t}
$$

where the best-fit value of $a$ is 2 (Bond 1978b). The ratio of feeding rates corresponding to deprivation for 8 $\mathrm{h}$ and $2 \mathrm{~h}$ can therefore be expressed as

$$
R=\frac{V_{8}}{V_{2}}=\frac{K-H(8)}{K-H(2)}
$$

Substituting for $H$ and collecting terms, we have

$$
\mathrm{R}=\frac{K\left(1-\frac{8}{a+8}\right)}{K\left(1-\frac{2}{a+2}\right)}
$$

Cancelling and evaluating at $a=2$, we find $R=$ $0.2 / 0.5=0.4$. According to Charnov's (1976a) hypothesis, the ratio of the giving-up times, $t_{\mathrm{g}^{\prime}}$ at the two deprivation levels should be equal to the inverse of the ratio of feeding rates, or $t_{\mathrm{g}}(2) / t_{\mathrm{g}}(8)=0.4$.

Because intensive searching in a uniform habitat intergrades smoothly with the subsequent extensive phase, it is difficult to extract a measure of giving-up time that does not also reflect the differences in the spatial distribution of foraging activity. The duration of searching within the innermost radial interval probably provides the best approximation to givingup time in the discrete model, since the effects of differences in spatial patterning of the search should be least apparent in close proximity to the point of prey contact. Within this interval, the ratio of search time in animals deprived for $2 \mathrm{~h}$ to animals deprived for $8 \mathrm{~h}$ was 0.46 , in general agreement with the theoretical prediction and in concurrence with several other measures of giving-up time obtained from lacewings searching on complex substrates (Bond 1978a). The uniformity of these measures implies that a consistent optimality criterion is being applied, even though the investment of foraging time in this case is the indirect result of temporal variation in the path parameters, rather than the expression of a simple decision to leave the patch.

While the evidence of optimality is reassuring, it must be noted that the applicability of the discrete 
theory in this case is very limited. If we had selected a larger radius, perhaps $2 \mathrm{~cm}$, for calculating $t_{\mathrm{g}}$, the ratio would have been closer to 0.6 than 0.4 , and the optimal distribution of searching effort would not have been apparent. The limited success of Charnov's theory is attributable in part to its having been designed to predict the temporal consequences of foraging behavior, rather than the mechanism by which those consequences are obtained. Such a theory may operate fairly well when it is applied to foraging on discrete patches, but it necessarily overlooks many of the most interesting features of the continuous case. One would like to know, for example, what optimality constraints determine the choice of the constant intercepts and asymptotes, what functions relate the decay constant and the velocity to the prey density, and what energetic effects are realized from the modifications in spatial distribution of searching effort. Some preliminary investigations along this line have been undertaken (Pyke 1978; Bond MS), but the most interesting aspects of the problem remain to be explored. Further analysis of foraging in uniform habitats awaits the development of a general theory of movement strategies, one that is capable of predicting the relative efficiency of various search mechanisms when applied over a range of possible prey densities and distributions.

\section{Acknowledgments}

I have been greatly assisted in this research by critical discussion and comments from G. W. Barlow, K. S. Hagen, R. L. Caldwell, S. J. Spengler and R. L. Tassan. Use of a digitizer was obtained through the kind offices of S. Dos Remedios, by permission of Kaiser Engineers. Support for this research was provided by a graduate fellowship from the National Institutes of Mental Health.

\section{References}

Bänsch, R. 1964. Vergleichende Untersuchungen zur Biologie und zum Beutefangverhalten aphidivorer Coccinelliden, Chrysopiden und Syrphiden. Zool. Jb. Syst. Bd., 91, 271-340.

Best, M. J. 1973. FCD: A feasible conjugate direction method to solve linearly constrained optimization problems. Waterloo, Canada: Department of Combinatorics and Optimization Research Report CORR 73-12, University of Waterloo.

Bond, A. B. MS. The efficiency of random searching.

Bond, A. B. 1978a. Hunger and foraging in the green lacewing, Chrysopa carnea Stephens. Diss. Abs. Int., 38, 4105-B. Ph.D. thesis, University of California, Berkeley, 1976.

Bond, A. B. 1978b. Food deprivation and the regulation of meal size in larvae of Chrysopa carnea. Physiol. Ent., 3, 27-32.

Chandler, A. E. F. 1969. Locomotory behaviour of first instar larvae of aphidophagous Syrphidae (Diptera) after contact with aphids. Anim. Behav., 17, 673-678.

Charnov, E. L. 1975. Optimal foraging: Some theoretical explorations. Diss. Abs. Int., 36, 2602-B. Ph.D. thesis, University of Washington, 1973.

Charnov, E. L. 1976a. Optimal foraging: the marginal value theorem. Theor. Popul. Biol., 9, 126-136.

Charnov, E. L. 1976b. Optimal foraging: attack strategy of a mantid. Am. Nat., 110, 141-151.

Cowie, R. J. 1977. Optimal foraging in great tits (Parus major). Nature, Lond., 268, 137-139.

Curio, E. 1976. The Ethology of Predation. Berlin: Springer-Verlag.

Fleschner, C. A. 1950. Studies on searching capacity of the larvae of three predators of the citrus red mite. Hilgardia, 20, 233-265.

Gibb, J. A. 1958. Predation by tits and squirrels on the eucosmid, Enarmonia eonicolana (Heyl). J. Anim. Ecol., 27, 376-396.

Holling, C. S. 1966. The functional response of invertebrate predators to prey density. Mem. Entomol. Soc. Can., 48, 1-86.

Jones, R. E. 1976. Search behaviour: A study of three caterpillar species. Behaviour, 60, 237-259.

Krebs, J. R. 1973. Behavioral aspects of predation. In: Perspectives in Ethology (Ed. P. P. G. Bateson \& P. H. Klopfer), pp. 73-111. New York: Plenum Press.

Krebs, J. R., Ryan, J., \& Charnov, E. L. 1974. Hunting by expectation or optimal foraging ? A study of patch use by chickadees. Anim. Behav., 22, 953-964.

Marascuilo, L. A. 1971. Statistical Methods for Behavioral Science Research. New York: McGraw-Hill.

Murdie, G. \& Hassell, M. P. 1973. Food distribution, searching success and predator-prey models. In: The Mathematical Theory of the Dynamics of Biological Populations (Ed. M. S. Bartlett \& R. W. Hiorns), pp. 87101. London: Academic Press.

Pyke, G. H. 1978. Are animals efficient harvesters? Anim. Behav., 26, 241-250.

Pyke, G. H., Pulliam, H. R., \& Charnov, E. L. 1977. Optimal foraging: A selective review of theory and tests. Q. Rev. Biol., 52, 137-154.

Smith, J. N. M. 1974a. The food searching behaviour of two European thrushes: 1. Description and analysis of the search paths. Behaviour, 48, 276-302. 
Smith, J. N. M. 1974b. The food searching behaviour of two European thrushes: II. The adaptiveness of the search patterns. Behaviour, 49, 1-61.

Smith, J. N. M. \& Sweatman, H. P. A. 1974. Food-searching behavior of titmice in patchy environments. Ecology, 55, 1216-1232.

Varley, G. C. 1941. On the search for hosts and the egg distribution of some chalcid parasites of the knapweed gall-fly. Parasitology, 33, 47-66.
Williamson, P. \& Gray, L. 1975. Foraging behavior of the starling (Sturnus vulgaris) in Maryland. Condor, 77, 84-89.

Wilson, D. S. 1976. Deducing the energy available in the environment: An application of optimal foraging theory. Biotropica, 8, 96-103.

Zach, R. \& Falls, J. B. 1976. Ovenbird (Aves: Parulidae) hunting behavior in a patchy environment: An experimental study. Can. J. Zool., 54, 1863-1879. 